ORIGINAL ARTICLE

\title{
Pediatric Urinary Tract Infection as a Cause of Outpatient Clinic Visits in Southern Ethiopia: A Cross Sectional Study
}

\author{
Enkosilassie Mitiku ${ }^{1}$, Anteneh Amsalu ${ }^{2}$, Birkneh Tilahun Tadesse ${ }^{3 *}$
}

\begin{abstract}
OPEN ACCESS
Citation: Enkosilassie Mitiku, Anteneh Amsalu, Birkneh Tilahun Tadesse. Pediatric Urinary Tract Infection as a Cause of Outpatient Clinic Visits in Southern Ethiopia: A Cross Sectional Study. Ethiop J Sci.2018;28(2):187. doi:http://dx.doi.org/10.4314/ejhs.v28i2.10 Received: October 11, 2017

Accepted: October 21, 2017

Published: March 1, 2018

Copyright: (C) 2018 Enkosilassie Mitiku., et al. This is an open access article distributed under the terms of the Creative Commons Attribution License, which permits unrestricted use, distribution, and reproduction in any medium, provided the original author and source are credited. Funding: Nil

Competing Interests: The authors declare that this manuscript was approved by all authors in its form and that no competing interest exists.

Affiliation and Correspondence:

${ }^{1}$ Department of Medical Microbiology Laboratory, Hawassa University Comprehensive Specialized Hospital, Hawassa, Ethiopia

${ }^{2}$ University of Gondar, Department of Microbiology

${ }^{3}$ College of Medicine and Health Sciences, Department of Pediatrics, Hawassa University, Ethiopia

*Email: birknehtilahun@gmail.com
\end{abstract}

BACKGROUND: Failure to timely diagnose and treat urinary tract infections is associated with grave long term consequences. The objectives of this study included assessing the proportion and predictors of Urinary Tract Infection (UTI) as a cause of pediatric outpatient department (OPD) visits and determining common uropathogens with antimicrobial susceptibility pattern.

METHODS: A cross sectional study was conducted from May to September 2015 among children of less than 15 years old at a tertiary center in Hawassa, Ethiopia. Children who fulfilled predefined eligibility criteria were recruited to undergo urine culture and urine analysis.

RESULTS: A total of 863 children visited the OPD during the study period among which $269(31.2 \%)$ fulfilled the predefined eligibility criteria. Urine culture was positive for 74/269(27.5\%) of the clinically suspected children. Male uncircumcision (adjusted odds ratio (aOR) 3.70; 95\% CI 1.34-10.16) and under nutrition (aOR 5.41; 95\%CI 2.64-11.07) were independent predictors of culture positivity. More than $5 \mathrm{WBC}$ per high power field (aOR 4.7, 95\% CI 1.8-12.7) on microscopy, urine $\mathrm{PH}>5.0$ (aOR 2.6, 95\%CI 1.2-5.8), and positive leukocyte esterase (aOR 9.9, 95\%CI 4.1-25.7) independently predicted positive growth on urine culture. Escherichia coli (34/74, 45.9\%) and Klebsiella spp (18/74, 24.3\%) were the most frequent isolates. High resistance was noted against amoxicillin (70.6\%) and cotrimoxazole (97.1\%) by E. coli.

CONCLUSION: UTI accounted for a tenth of total OPD visits. Commonly used first line antibiotics showed high level resistance to common etiologies of UTI. UTI should be suspected in febrile children, and antibiograms should be done to tailor prescription of antibiotics.

KEYWORDS: Bacterial profile, antibiotic resistance, children, urinary tract infection

\section{INTRODUCTION}

Urinary Tract Infection (UTI) is the most common childhood bacterial infection associated with high morbidity and long-term complications like renal scarring, hypertension, and chronic renal failure (1). To reduce the adulthood complications, early diagnosis, 
proper investigation, adequate

therapy and prolonged follow-up are of outmost importance.

The epidemiology of UTI during childhood varies by age, gender, circumcision status and other factors. Boys are more susceptible during the first year of life, mostly the first 3 months; thereafter the incidence is substantially higher in girls (2). Among boys, uncircumcised infants have an eightfold higher risk (3). About 5\% of girls and 2\% of boys experience at least one episode of UTI up to the age of seven years (4). Clinically, important UTIs usually occur due to bacteria, although viruses, fungi, and parasites can also cause infection (5). Gram negative enteric bacilli such as Escherichia coli, Klebsiella ssp., Proteus ssp., Enterobacter ssp., Pseudomonas ssp., and Citrobacter spp. and Gram-positive organisms, including group B streptococci, Enterococcus spp, and Staphylococcus aureus are commonly associated with pediatric UTI $(1,4,5)$.

Effective management of patients suffering from severe UTI commonly relies on the identification of pathogenic organisms and the selection of an effective antibiotic agent to the organism in question. However, diagnosis of UTI is often delayed due to obscure clinical findings, particularly in infants and children of less than tow years $(2,3,6)$. Moreover, it is difficult to collect urine and interpret the results to confirm the diagnosis unequivocally in this age group (3). Hence, empirical antibiotic prescription is often endorsed even without culture and sensitivity. On the other hand, the emergence of antibiotic resistant uropathogens in pediatric urology is increasing worldwide; particularly in developing countries where empirical treatment is the mainstay of treatment in the absence of proper diagnostic modalities and the availability of antibiotics over the counter $(7,8)$.

The aqnibiotic susceptibility profile of uropathogens is variable and usually depends on time, geographical location, demography and clinical characteristics of patients (9). An updated information in a specific location may aid clinicians in choosing the appropriate antibiotic for empirical treatment. However, shortage of data regarding the antibiogram of commonly encountered uropathogens, empirical antibiotic treatments following the WHO empiric guidelines is the common practice in the study setting.

Given the prescription practices following the WHO empiric guidelines in the study setting, understanding the common etiologies of pediatric UTI and their antibiograms would assist clinicians to optimize treatment strategies for such patients. This study tries to address three important questions:

1. How common is UTI as a cause of OPD visits?

2. What are the common etiologies of UTI in the study setting?

3. What is the antibiotic susceptibility profile of pediatric uropathogens?

\section{METHODS}

Study setting: A hospital based cross-sectional study was conducted from May to September 2015 at the Outpatient Department (OPD) of Hawassa University Comprehensive Specialized Hospital (HUCSH) in Southern Ethiopia. The hospital serves as both primary care and referral teaching hospital for both Southern Nations Nationalities and Peoples Region (SNNR) and Oromia regions. It is located $275 \mathrm{~km}$ south of the capital city, Addis Ababa.

Eligibility criteria: We recruited children of less than 15 years of age who were attending at the the pediatric OPD of HUCSH. For enrolment to the study, we followed predefined inclusion criteria which were adapted from Grabe et al, $2015(2,10)$. We included children who had one or more of the following:

1) Fever which was defined as axillary temperature of $37.5{ }^{\circ} \mathrm{C}$ or higher at presentation;

2) At least two episodes of vomiting within two days of presentation at OPD;

3) Dysuria as complained by older children or crying during urination for young children as reported by the mother;

4) Urinary frequency which is defined as passing urine more frequently than the previous normal for the child; 
5) Urinary urgency as complained in older children;

6) Loin pain/tenderness or urinary color change.

We excluded children who had taken antibiotics within the last two weeks and if mothers or the child refused to give consent for participation. A sample size of 192 children was calculated based on the assumptions of a $25 \%$ culture positive rate according to Ali E and Osman H (10), power of $80 \%$, with $95 \%$ confidence interval and $5 \%$ margin of error and 10\% non-response rate. A study period was decided based on the predefined patient flow and the sample size. However, we have ultimately taken all the children who fulfilled the inclusion criteria during the study period.

Data collection: After obtaining informed written consent from parents/guardians and assent from older children, socio-demographic and clinical data were collected using structured questionnaires. Nutritional status, weight and height of children were assessed by trained nurses and clinical examination was carried out by the attending physician. For patients who fulfilled the inclusion criteria, midstream urine sample was collected in a wide mouthed sterile container after cleansing the urethral meatus after thoroughly instructing their guardians. The collected urine sample was delivered to the hospital microbiology laboratory within 30 minutes.

Urine culture: All urine specimens were inoculated into blood agar and MacConkey agar plate (Oxide, Ltd., Basingstoke, and Hampshire, England), using a calibrated loops $(0.002 \mathrm{ml})$ for the semi-quantitative method. Streaked culture plates were incubated at $37^{\circ} \mathrm{c}$ overnight. More than $10^{5}$ colony forming units per milliliter of urine $(\mathrm{cfu} / \mathrm{mL})$ and growth of a single organism was considered as culture positive to further decrease the possibility of contamination. Pure isolates of bacterial pathogen were preliminary characterized by colony morphology and Gramstain. All positive urine cultures showing significant bacteriuria were further identified by their characteristic appearance on their respective media and confirmed by the pattern of biochemical reaction using the standard procedures (11). Urine culture was done by experienced graduate medical microbiology students under the close supervision of experienced microbiologists.

Antibiotic susceptibility test: The antibiotic susceptibility test was performed by Kirby-Bauer disk diffusion method (12). In brief, pure culture was transferred into a tube containing $5 \mathrm{ml}$ nutrient broth and mixed gently until it forms a homogenous suspension. The turbidity of the suspension was compared with 0.5 McFarland standards in order to standardize the inoculums density. A sterile cotton swab was dipped into the suspension and the excess were removed by gentle rotation of the swab against the surface of the tube. The swab was then used to distribute the bacteria suspension evenly over the entire surface of Mueller-Hinton agar (Oxoid). The inoculated plates were left at room temperature to dry for 3-5 minutes. The antibiotics for disc diffusion testing were obtained from Oxoid that include: ciprofloxacin $(5 \mu \mathrm{g})$, norfloxacilin $(10 \mu \mathrm{g})$, nitrofurantoin $(300 \mu \mathrm{g})$, ceftriaxone $(30 \mu \mathrm{g})$, cefotaxime $(30 \mu \mathrm{g})$, cefazolin $(30 \mu \mathrm{g})$, gentamicin $(10 \mu \mathrm{g})$, co-trimoxazole $(1.25 / 23.75 \mu \mathrm{g})$, ampicillin $(10 \mu \mathrm{g})$, cefoxitin $(30 \mu \mathrm{g})$, penicillin $\mathrm{G}(10 \mathrm{IU})$ and erythromycin $(15 \mu \mathrm{g})$. After 18-24 hours incubation at $37^{\circ} \mathrm{C}$, zone of growth inhibition was measured to the nearest whole millimeter using a caliper. The zone diameters were interpreted according to the Clinical and Laboratory Standards Institute (CLSI) guideline (13) as susceptible (S), intermediate (I) or resistant (R).

Quality control: The completeness of the questionnaire was verified by the principal investigator daily. Sterility of culture media and biochemical tests were checked by overnight incubation of un-inoculated media from each batch of preparation. Standard strains of E. coli ATCC 25922, P. aeruginosa ATCC 27853 and $S$. aureus ATCC 25923 were used during culture and antibiotic susceptibility testing for internal quality assurance.

Data analysis: Data were coded, entered and analyzed using $\mathrm{R}$ software version 3.3.2. Data were presented as percentage, mean (standard deviation (SD)) and median (range). Differences

DOI: http://dx.doi.org/10.4314/ejhs.v28i2.10 
in proportions were evaluated by Pearson's chisquare and Fisher exact tests; $p<0.05$ was considered to be significant. Bivariate and multivariate logistic regression were used to determine the predictors of culture proven UTI. Adjusted odds ratio (aOR) with 95\% CI was also computed using multivariable logistic regression analysis, taking all factors yielding a p-value $\leq$ 0.05 in bivariate analysis for adjusting possible confounders. In this study, undernutrition is defined as having a weight-for-height/length (WFH) below-2SD based on the WHO growth standards (14).

Ethics approval and consent to participate: Ethical approval was obtained from Hawassa University, College of Medicine and Health Sciences, Institutional Review Board (IRB). A permission letter was obtained from the hospital administration. Informed written consent and/or assent were obtained from children and/or guardians. Patient information was anonymized and de-identified prior to analysis. Culture results were given to the physician, and patients were treated accordingly.

\section{RESULTS}

From May 1, 2015 to September 30, 2015, a total of 269/863(31.2\%) children fulfilled the inclusion criteria for clinically suspected UTI. Two-third $(61.7 \%)$ of the children were male. The mean age of the participants was $4.8(\mathrm{SD}=3.6)$ years. The majority $(195 / 269,72.5 \%)$ presented with fever; whereas $\quad 81 / 269(31.1 \%), \quad 92 / 269(34.2 \%)$, $33 / 269(12.3 \%)$, and $33 / 269(12.3 \%)$ presented with dysuria, vomiting, urinary frequency and lower abdominal pain respectively. More than half $(149 / 269,55.4 \%)$ were rural residents, $31 / 269(11.5 \%)$ had any degree of under nutrition (mild moderate or severe) and $8 / 269(3.0 \%)$ of the children had diabetes mellitus. Previous history of UTI was reported in 20/269(7.4\%) of the children, and $71 / 269(26.4 \%)$ had taken any antibiotics in the last three months prior to current presentation (Table 1). None of the subjects reported any history of previous renal problems.
Table 1: General characteristics of children who had clinical signs and symptoms suggestive of UTI at the OPD of Hawassa University Comprehensive Specialized Hospital, 2015.

\begin{tabular}{|c|c|}
\hline Variable (total 269) & Frequency(\%) \\
\hline \multicolumn{2}{|l|}{ Age (years) } \\
\hline$<1$ & $54(20.5)$ \\
\hline $1-5$ & $107(39.8)$ \\
\hline $6-14$ & $108(40.1)$ \\
\hline \multicolumn{2}{|l|}{ Sex } \\
\hline Female & $103(38.3)$ \\
\hline Male & $166(61.7)$ \\
\hline \multirow{2}{*}{\multicolumn{2}{|c|}{$\begin{array}{l}\text { Circumcision in boys } \\
(166)\end{array}$}} \\
\hline$(166)$ & \\
\hline Yes & $119(71.7)$ \\
\hline No & \\
\hline \multicolumn{2}{|l|}{ Residence } \\
\hline Rural & $120(44.6)$ \\
\hline Urban & $149(55.4)$ \\
\hline \multicolumn{2}{|l|}{ Previous antibiotic intake } \\
\hline No & $16(5.9)$ \\
\hline Unknown & $182(67.7)$ \\
\hline Yes & $71(26.4)$ \\
\hline \multicolumn{2}{|l|}{ Previous history of UTI } \\
\hline Yes & $20(7.4)$ \\
\hline No & $208(77.3)$ \\
\hline Unknown & $41(15.2)$ \\
\hline \multicolumn{2}{|l|}{$\begin{array}{l}\text { Clinical presentation at the } \\
\text { time of diagnosis }\end{array}$} \\
\hline Fever & $195(72.5)$ \\
\hline Dysuria & $81(31.1)$ \\
\hline Vomiting & $92(34.2)$ \\
\hline Urinary frequency & $33(12.3)$ \\
\hline $\begin{array}{l}\text { Lower abdominal } \\
\text { pain }\end{array}$ & $33(12.3)$ \\
\hline \multicolumn{2}{|l|}{ Under-nutrition } \\
\hline Yes & $31(11.5)$ \\
\hline No & $238(88.5)$ \\
\hline \multicolumn{2}{|l|}{ Diabetes mellitus } \\
\hline Yes & $8(3.0)$ \\
\hline No & $261(97.0)$ \\
\hline
\end{tabular}

UTI: urinary tract infection 
Bacterial isolation: Midstream clean catch urine culture was positive for $74 / 269(27.5 \%)$ of the patients who had suspected UTI. Overall, UTI contributed for $74 / 863(8.6 \%)$ of the total pediatric OPD visits in five months. Males had a slightly higher isolation rate of $40 / 74(54.5 \%)(p$ value $=0.147)$. Among males, $119(71.7 \%)$ were not circumcised; and 36/40(90.05) of the boys who had a positive urine culture were not circumcised $\left(X^{2}=7.6, \quad p\right.$-value $\left.=0.006\right)$. The majority $(61 / 74,82.4 \%)$ of the isolates were Gram negative bacteria. E. coli $(36 / 74,48.6 \%)$ was the most frequent isolate followed by Klebsiella spp. (18/74, 24.3\%); S. aureus (7/74, 9.5\%);
Enterococcus spp. (6/74, 8.1\%); Citrobacter spp.(4/74, 5.4\%); Enterobacter spp. (2/74, 2.7\%); Proteusspp. (2/74, 2.7\%), and Salmonella spp. $(1 / 74,1.4 \%)$. Isolation of $E$. coli was significantly more frequent in females $(25 / 36,69.4 \%)$ than in males $\quad(11 / 36, \quad 30.6 \%) \quad\left(X^{2}=15.6, \quad p\right.$ value $<0.0001)$. On the other hand, all the $7(100 \%)$ of the $S$. aureus isolates ( $p$-value $=0.04)$, and the majority $(15 / 18,83.3 \%)$ of Klebsiella spp, though statistically insignificant, were isolated from boys $\left(X^{2}=2.9, \quad p\right.$-value $\left.=0.089\right)$. Culture positivity of urine culture was much higher for children who presented with fever, dysuria and vomiting $\left(X^{2}=33.53, d f=8, p\right.$-value $\left.<0.0001\right)$ (Figure 1).

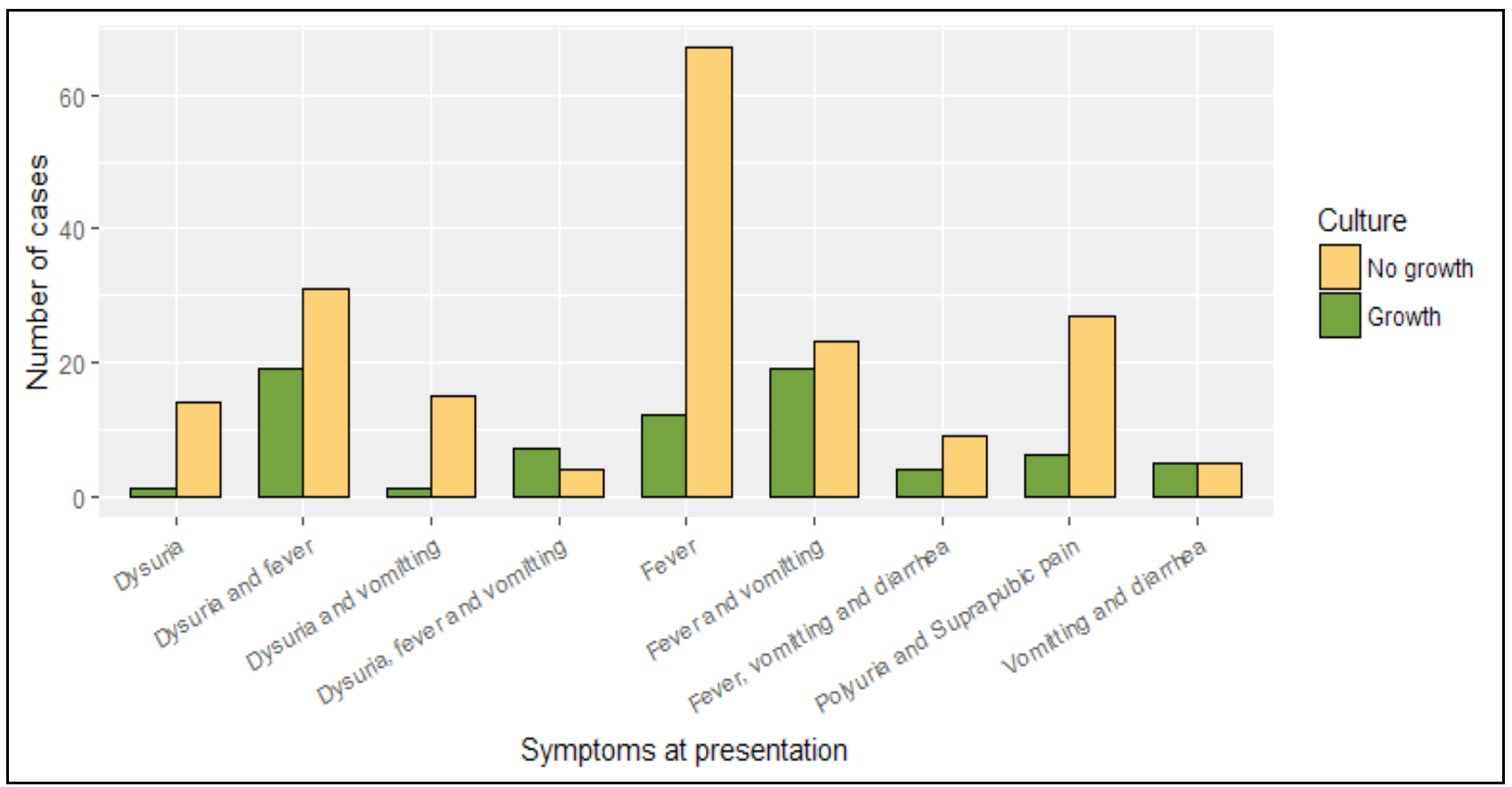

Figure 1: Number cases of positive and negative culture result based on symptoms at presentation to the hospital from May 2015 to September 2015

Predictors of culture confirmed UTI: Rural residence (aOR 2.84, 95\% CI 1.74-4.64), any degree of under nutrition (aOR 5.41, 95\%CI 2.64$11.07)$ and absence of circumcision in boys (aOR $3.70,95 \%$ CI 1.34-10.16) have been found to be independent predictors of culture confirmed UTI. However, on including circumcision in the multivariate regression model, we observed that residence is no more an independent predictor (aOR 0.97, 95\% CI 0.46-2.03) (Table 2). Further looking into the relationship between residence and circumcision, it is noted that rural residents are more likely to be uncircumcised $\left(X^{2}=40.41\right.$, $\mathrm{df}=1$, $\mathrm{p}$-value $<0.0001$ ). 
Table 2: Predictors of urinary tract infection among children who visited pediatric outpatient department of Hawassa University Comprehensive Specialized Hospital between May and September 2015.

\begin{tabular}{|c|c|c|c|c|c|}
\hline $\begin{array}{l}\text { Variable } \\
\text { (total patients) }\end{array}$ & $\begin{array}{l}\text { No UTI } \\
\text { N }(\%)\end{array}$ & $\begin{array}{l}\text { UTI } \\
\text { N (\%) }\end{array}$ & $\begin{array}{l}p- \\
\text { value }\end{array}$ & $\begin{array}{l}\text { Crude OR(95\% } \\
\text { CI) }\end{array}$ & $\begin{array}{l}\text { Adjusted OR } \\
(95 \% \text { CI) }\end{array}$ \\
\hline Age, mean(SD) $(n=269)$ & $4.85(3.88)$ & $4.78(3.53)$ & 0.903 & $1.01(0.93-1.08)$ & \\
\hline $\operatorname{Sex}(n=269)$ & & & 0.113 & & \\
\hline Male & $69(67.0)$ & $34(33.0)$ & 1 & 1 & \\
\hline Female & $126(75.9)$ & $40(24.1)$ & 0.113 & $0.64(0.41-1.02)$ & \\
\hline \multicolumn{6}{|l|}{ Residence $(n=269)$} \\
\hline Rural & $95(63.8)$ & $54(36.2)$ & 0.000 & $2.84(1.74-4.64) *$ & $0.97(0.46-2.03)$ \\
\hline Urban & $100(83.3)$ & $20(16.7)$ & 1 & 1 & 1 \\
\hline \multicolumn{6}{|l|}{$\begin{array}{l}\text { Previous history of UTI } \\
(\mathrm{n}=269)\end{array}$} \\
\hline No & $152(73.1)$ & $56(26.9)$ & 1 & 1 & \\
\hline Unknown & $30(73.2)$ & $11(26.8)$ & 0.990 & $1.0(0.53-1.88)$ & \\
\hline Yes & $13(65.0)$ & $7(53.0)$ & 0.443 & $1.46(0.65-3.29)$ & \\
\hline \multicolumn{6}{|l|}{ Under-nutrition $(n=269)$} \\
\hline Yes & $10(32.3)$ & $21(67.7)$ & 0.000 & $7.33(3.71-14.50)^{*}$ & $5.41(2.64-11.07)$ \\
\hline No & $185(77.7)$ & $53(22.3)$ & 1 & 1 & 1 \\
\hline \multicolumn{6}{|l|}{$\begin{array}{l}\text { Previous antibiotic intake } \\
(\mathrm{n}=269)\end{array}$} \\
\hline No & $54(29.7)$ & $128(70.3)$ & 1 & 1 & \\
\hline Unknown & $5(31.2)$ & $11(68.8)$ & 0.985 & $1.07(0.43-0.55)$ & \\
\hline Yes & $15(21.1)$ & $56(78.9)$ & 0.172 & $0.63(0.37-1.10)$ & \\
\hline \multicolumn{6}{|l|}{$\begin{array}{l}\text { Previous hospital } \\
\text { admission }(n=269)\end{array}$} \\
\hline Yes & $20(62.5)$ & $12(37.5)$ & 0.181 & $0.59(0.31-1.13)$ & \\
\hline No & $175(73.8)$ & $62(26.2)$ & 1 & 1 & \\
\hline \multicolumn{6}{|l|}{$\begin{array}{l}\text { Circumcision in boys } \\
(\mathrm{n}=166)\end{array}$} \\
\hline Yes & $43(91.5)$ & $4(8.5)$ & 1 & 1 & 1 \\
\hline No & $83(69.7)$ & $36(30.3)$ & 0.006 & $4.66(2.00-13.02)^{*}$ & $3.70(1.34-10.16)$ \\
\hline
\end{tabular}

UTI: urinary tract infection, ${ }^{*}$ p-value $:<0.05$

All the patients underwent urine microscopy and dipstick. The findings of more than five white blood cells (WBC) per high power field (HPF) (aOR 4.7, 95\% CI 1.8-12.7) on urine microscopy, urine $\mathrm{PH}$ higher than 5.0 (aOR 2.6, 95\%CI 1.25.8), and a positive leukocyte esterase (aOR 9.9,
95\% CI 4.1-25.7) independently predicted a higher chance of bacterial isolation on urine culture. Moreover, all the four patients who had a positive nitrite test had a positive urine culture (Table 3). 
Table 3: Urine analysis findings and their association with urine culture positivity among children seen at OPD of Hawassa University Comprehensive Specialized Hospital, 2015.

\begin{tabular}{|c|c|c|c|c|c|}
\hline \multirow[t]{2}{*}{ Variable } & \multicolumn{2}{|c|}{ Urine Culture } & \multirow[t]{2}{*}{ P-value } & \multirow{2}{*}{$\begin{array}{l}\text { Crude OR (95\% } \\
\text { CI) }\end{array}$} & \multirow{2}{*}{$\begin{array}{l}\text { Adjusted OR } \\
(95 \% \text { CI) }\end{array}$} \\
\hline & No $(\%)$ & Yes $(\%)$ & & & \\
\hline White Blood Cell (per HPF) & & & $<0.0001$ & & \\
\hline Less than 5 cells & $152(93.3)$ & $11(6.7)$ & & 1 & 1 \\
\hline More than 5 cells & $43(40.6)$ & $63(59.4)$ & & $20.2(10.2-43.7)^{*}$ & $4.7(1.8-12.7)$ \\
\hline Urine PH & & & 0.0005 & & \\
\hline PH less than 5.0 & $120(81.1)$ & $28(18.9)$ & & 1 & 1 \\
\hline PH 5.0 and above & $75(62.0)$ & $46(38.0)$ & & $2.6(1.5-4.6) *$ & $2.6(1.2-5.8)$ \\
\hline Urine Specific Gravity & & & 0.975 & & \\
\hline $1.0-1.015$ & $61(72.6)$ & $23(27.4)$ & & 1 & - \\
\hline $1.020-1.030$ & $134(72.4)$ & $51(27.6)$ & & $1.0(0.6-1.8)$ & \\
\hline Red Blood Cells (per HPF) & & & 0.123 & & \\
\hline Less than 5 cells & $168(74.3)$ & $58(25.7)$ & & 1 & - \\
\hline More than 5 cells & $27(62.8)$ & $16(37.2)$ & & $1.7(0.9-3.0)$ & \\
\hline Cast & & & $<0.0001$ & & \\
\hline No cast & $189(77.5)$ & $55(22.5)$ & & 1 & 1 \\
\hline Cellular or Granular & $6(24.0)$ & $19(76.0)$ & & $10.9(5.0-25.9)^{*}$ & $2.5(0.7-9.5)$ \\
\hline Nitrite & & & $0.006 \dagger$ & & \\
\hline Negative & $195(73.6)$ & $70(26.4)$ & & & \\
\hline Positive & 0 & $4(100)$ & & & \\
\hline Leukocyte Esterase & & & $<0.0001$ & & \\
\hline Negative & $180(89.6)$ & $21(10.4)$ & & 1 & 1 \\
\hline Positive & $15(22.1)$ & $53(77.9)$ & & $30.3(15.0-64.9)^{*}$ & $9.9(4.1-25.7)$ \\
\hline Urinary Crystals & & & $0.437 \dagger$ & & \\
\hline None & $190(73.1)$ & $70(26.9)$ & & & \\
\hline Yes & $5(55.6)$ & $4(44.4)$ & & & \\
\hline Urine protein & & & & & \\
\hline None & $172(76.4)$ & $53(23.6)$ & 1 & 1 & 1 \\
\hline Trace & $14(70.0)$ & $6(30.0)$ & 0.519 & $1.4(0.5-3.7)$ & $1.4(0.3-5.1)$ \\
\hline More than $1+$ & $9(37.5)$ & $15(62.5)$ & 0.0002 & $5.4(2.3-13.6)^{*}$ & $1.5(0.4-5.4)$ \\
\hline Urine Glucose & & & $0.899 \dagger$ & & \\
\hline None & $192(72.7)$ & $72(27.3)$ & & & \\
\hline Trace and above & $3(60.0)$ & $2(40.0)$ & & & \\
\hline
\end{tabular}

HPF: high power filed, * $p$-value $<0.05$; †bivariate logistic regression was not done as there are zero or few cases in a category.

Antibiogram of isolates: All the E. coli isolatesthe commonest cause of UTI in children-were resistant to ampicillin, penicillin and oxacillin, while the majority $(97.1 \%)$ were resistant to cotrimoxazole, which is the first line drug for pediatric uncomplicated UTI. On the other hand, resistance of $E$. coli to nitrofurantoin $(0 \%)$ and quinolones $(11.8 \%)$ was the lowest of all the tested antibiotics. Gentamicin resistance to Klebsiella spp, which is naturally resistant to ampicillin, was observed to be high $(66.7 \%)$ (Table 4). 
Table 4: Antimicrobial resistance pattern of bacterial isolates described as number of resistant isolates over total number of isolates tested for the specific antibiotic among pediatric UTI patients at Hawassa University Comprehensive Specialized Hospital, 2015

\begin{tabular}{lccccccc}
\hline Antibiotic & $\begin{array}{c}\text { E. coli } \\
(\mathbf{n}=\mathbf{3 4})\end{array}$ & $\begin{array}{c}\text { Klebsiella } \\
\mathbf{s p p .}\end{array}$ & $\begin{array}{c}\text { Citrobacter } \\
\text { spp. }(\mathbf{n}=\mathbf{3})\end{array}$ & $\begin{array}{c}\text { Enterobacter } \\
\text { spp. }(\mathbf{n}=\mathbf{2})\end{array}$ & $\begin{array}{c}\text { Salmonella } \\
\text { spp. }(\mathbf{n}=\mathbf{1})\end{array}$ & $\begin{array}{c}\text { S. } \\
\text { aureus } \\
(\mathbf{n}=7)\end{array}$ & $\begin{array}{c}\text { Enterococcus } \\
\text { spp.(n=6) }\end{array}$ \\
\hline Ampicillin & 100 & 88.9 & 100 & 100 & 0 & 85.7 & 83.3 \\
Amoxicillin & 70.6 & 77.8 & 100 & 100 & 0 & 14.3 & 33.3 \\
Cotrimoxazole & 97.1 & 94.4 & 100 & 100 & 100 & 14.3 & 16.7 \\
Gentamicin & 41.2 & 66.7 & 66.7 & 0 & 0 & 57.1 & 50 \\
Ciprofloxacin & 11.8 & 44.4 & 0 & 0 & 0 & 57.1 & 50 \\
Norfloxacin & 23.5 & 11.1 & 0 & 0 & 0 & 71.4 & 50 \\
Ceftriaxone & 47.1 & 38.9 & 66.7 & 0 & 0 & 71.4 & 50 \\
Cephalexin & 44.1 & 72.2 & 0 & 100 & 0 & 0 & 0 \\
Cefotaxime & 29.4 & 16.7 & 33.3 & 0 & 0 & 0 & 16.7 \\
Nitrofurantoin & 0 & 22.2 & 0 & 50 & 0 & 71.4 & 50 \\
Oxacillin & 100 & 100 & 100 & 100 & 100 & 42.9 & 83.3 \\
Penicillin G & 100 & 100 & - & - & - & 85.7 & 66.7 \\
Erythromycin & - & 100 & - & - & - & 71.4 & 50 \\
\hline
\end{tabular}

\section{DISCUSSION}

Although UTI causes significant illness in children, the definitive diagnosises of UTI in most developing countries are often overlooked due to lack of facilities and laboratory cost in addition to difficulties of obtaining urine from children especially those who would not void voluntarily $(6,15,16)$. In this study, culture confirmed UTI among clinical suspected children was $27.5 \%$ which is concordant with a study conducted in India $(32.2 \%)$ but higher than a study reported in Iran $(3.6 \%)(17,18)$. The differences could be explained by the retrospective study design they used and other demographic differences such as the majority of study subjects being females in contrast to our study. Moderate and severe degrees of undernutrition were independently associated with higher risk of UTI which could be explained by immunosuppression associated with undernutrition $(2,19)$. Additionally, the finding of significantly more boys with uncircumcision from rural settings could explain the higher isolation.

In this study, E. coli and Klebsiella spp. accounted for about three fourth of the total isolates. The presence of unique structures such as adhesion, pili, fimbriae and P1-blood group phenotype receptor in Enterobacteriaceae which help for their attachment to the uroepithelium increases risk for infection (20). We found a relatively higher rate of isolation of Klebsiella spp. and $S$. aureus as compared to previous reports $(1,15,18)$. E. coli, which is the most common pathogen in both sexes, was significantly more frequently isolated in females than in males which is congruent with previous studies $(21,22)$. On the other hand, similar with our findings, isolation rates of Klebsiella spp. and $S$. aureus have been reported to be higher in males than females $(21,22)$. The differences in the preputial flora have been proposed as the possible reasons for differences in isolation rates (23). Moreover, findings of higher frequency of dilating vesicoureteral reflux (VUR) in boys (24) could be the explanation for the differences $(22,25)$.

The more antibiotics are used in a community, the more likely it is that resistant strains will be selected and maintained in the environment $(8,15,26)$. This complements the current high rate of resistance following ampicillin, co-trimoxazole, gentamicin and ceftriaxone exposure within three months prior to the diagnosis of UTI in our hospital. High resistance to these antibiotics is perhaps due to their widespread and erroneous use as empirical therapy even when guidelines suggest that when resistance rate exceeds $20 \%$, the drug should not be

DOI: http://dx.doi.org/10.4314/ejhs.v28i2.10 
used empirically (27). In this study, rates of antimicrobial resistance profile of $E$. coli to ampicillin were high similar to studies from another African setting (15) but higher than Asian (79.3\%) (28) and European settings(50\%)(29). Our finding of high resistance profile of the common etiologies of UTI to the first line antibiotics is high and calls for more studies in this area in order to revise guidelines.

In this study, nitrofurantoin showed low level of resistance which makes this drug a reasonable alternative in management of UTI. This might have been due to its multiple mechanisms of action (1), underusage and narrow-spectrum nature of the drug. However, it cannot be used to treat UTI in complicated and febrile infants because it is excreted in the urine and does not achieve therapeutic concentrations in the bloodstream (30).

This study tried to address important research questions regarding the contribution of UTI for pediatric OPD visits, rate of isolation of bacterial causes among suspected cases based on signs and symptoms and the predictors of a culture confirmed UTI. It also revealed the antimicrobial resistance pattern of clinically relevant pathogens that caused UTI. However, it also had limitations including being hospital/suspicion based study where less severe or asymptomatic cases of UTI might have been missed further limiting its generalizability to the whole pediatric population. It also involved a small sample size especially for antimicrobial testing which limits the recommendations for revising treatment guidelines. Moreover, even though we took measures to minimize any risk of contamination, for younger age groups, the urine collection mechanism we have used might have increased the risk of contamination.

In conclusion, clinically suspected UTI accounted for nearly a third of the total OPD visits, and around a third of these were culture confirmed UTI. Moderate and sever degree of undernutrition and male uncircumcision were associated with increased risks of culture confirmed UTI. The commonly used first line antibiotics like amoxicillin, ampicillin and cotrimoxazole showed a high level resistance limiting their use for OPD treatment of UTIs. The findings of the current study call for a more robust study with a larger isolate number to better understand the resistance pattern of the common bacterial isolates and advise guideline change.

\section{ACKNOWLEDGEMENTS}

We are most grateful to express our gratitude to staff members of pediatric and microbiology laboratory department for their professional assistance during the data collection and analysis.

\section{REFERENCES}

1. Echeverri CV, Serna-HiguitaLM, Serrano AK, Ochoa-García C, Rosas LR, Bedoya AM, et al. Resistance profile for pathogens causing urinary tract infection in a pediatric population, and antibiotic treatment response, at a University Hospital 2010-2011. Colomb Med 2014; 45(1):39.

2. Grabe M, Bartoletti R, Bjerklund JTE, et al. Guidelines on Urological Infections. EAU 2015.

3. Kibar Y. Current Management of Urinary Tract Infection in Children, Urinary Tract Infections, Dr. Peter Tenke (Ed.), ISBN: 978-953-307-757-4, InTech. 2011:Available from: http://www.intechopen.com/books/urinary-tractinfections/current-management-of-urinary-tractinfection-inchildren.

4. Habib S. Highlights for management of a child with a urinary tract infection. Int J Pediatr 2012; 2012:1-6.

5. Zorc JJ, Kiddoo DA, Shaw KN. Diagnosis and Management of Pediatric Urinary Tract Infections. Clin Microbio Reviews 2005; 18(2):417-422.

6. Ayazi P, Mahyar A, Hashemi HJ, Khabiri S. Urinary Tract Infections in Children. Iranian $J$ Pediatr Soc 2010; 2(1):9-14.

7. Bryce A, Hay AD, Lane IF, Thornton HV, Wootton M, Costelloe C. Global prevalence of antibiotic resistance in paediatric urinary tract infections caused by Escherichia coli and association with routine use of antibiotics in primary care: systematic review and metaanalysis. $B M J \quad 2016 ; \quad 352:$ i939; doi.org/910.1136/bmj.i1939.

8. Edlin RS, Copp HL. Antibiotic resistance in pediatric urology. Ther Adv Urol 2014; 6(2):5461: DOI: $10.1177 / 1756287213511508$.

9. Shaikh N, Morone NE, Bost JE, Farrell MH. Prevalence of Urinary Tract Infection in Childhood A Meta-Analysis. Pediatr Infect Dis $J$ 2008; 27:302-308.

10. Eltigani MA and Hassan A. OsmanbAcute Urinary Tract Infections in Children in Khartoum State:

DOI: http://dx.doi.org/10.4314/ejhs.v28i2.10 
Pathogens, Antimicrobial Susceptibility and Associated Risk Factors. Arab Journal of Nephrology and Transplantation. 2009 May;2(2):11-5.

11. Cheesbrough M. District Laboratory Practical in Tropical Countries, 2ndedition, part (2). Cambridge University press Cambridge, New York. 2006.

12. Bauer AW, Kirby WMM, Sherris JC, Turck M. Antibiotic susceptibility testing by standard single disc method. Am J Clin Pathol 1966; 45:493-496.

13. CLSI: Performance standards for antimicrobial susceptibility testing; twenty-second information supplement. CLSI document M100-S22. Wayne, PA: Clinical and Laboratory Standards Institute. 2012.

14. WHO Multicentre Growth Reference Study Group: WHO child growth standards: methods and development. Growth velocity based on weight, length and head circumference. Geneva, World Health Organization. 2009.

15. Adjei O, Opoku C. Urinary tract infections in African infants. Int $J$ Antimicrob Agents 2004; 1:32-34.

16. Patel P, Garala RN. Bacteriological profile and antibiotic susceptibility pattern (antibiogram) of urinary tract infections in paediatric patients. $J$ of Resear in Medical and Dental Sci 2014; 2(1).

17. Fredrick F, Francis J, Fataki M, Maselle S. A etiology, antimicrobial susceptibility and predictors of urinary tract infection among febrile under-fives at Muhimbili National Hospital, Dares Salaam-Tanzania. African J of Microbio Res 2013; 12:1029-1034.

18. Rezaee MA, Abdinia B. Etiology and Antimicrobial Susceptibility Pattern of Pathogenic Bacteria in Children Subjected to UTI. Medicine 2015; 94(39):e1606; doi: 1610.1097/MD.0000000000001606.

19. Çaksen H, Cesur Y, Üner A, Arslan S, Sar S, Çelebi V, Kuru M. Urinary tract infection and antibiotic susceptibility in malnourished children. Int Urol and Nephrol 2000; 32:245.

20. Das RN, Chandrashekhar TS, Joshi HS GM, Shrestha N, Shivananda PG. Frequency and susceptibility profile of pathogens causing urinary tract infections at a tertiary care hospital in western Nepal. Singapore Med J 2006; 47(4):281.

21. Mirsoleymani RS, Salimi M, Brojeni MS, Ranjbar M, Mehtarpoor M. Bacterial Pathogens and Antimicrobial Resistance Patterns in Pediatric
Urinary Tract Infections: A Four-Year Surveillance Study (2009-2012). Int J of Pediatr 2014; 2014:1-6: doi.org/10.1155/2014/126142.

22. Swerkersson S, Jodal U, Åhrén C, Hansson S. Urinary tract infection in small outpatient children: the influence of age and gender on resistance to oral antimicrobials. Eur J Pediatr 2014; 173:1075.

23. Prelog M, Schiefecker D, Fille M, Wurzner R, Brunner A, Zimmerhackl LB. Febrile urinary tract infection in children: ampicillin and trimethoprim insufficient as empirical mono-therapy. Pediatr Nephrol 2008; 23:597-602.

24. Kanellopoulos TA, Salakos C, Spiliopoulou I, Ellina A, Nikolakopoulou NM, Papanastasiou DA. First urinary tract infection in neonates, infants and young children: a comparative study. Pediatr Nephrol 2006; 21:1131-1137.

25. Becknell B, Schober M, Korbel L, David Spencer J. The Diagnosis, Evaluation and Treatment of Acute and Recurrent Pediatric Urinary Tract Infections. Expert Rev Anti Infect Ther 2015;13 (1):81-90. doi:10.1586/14787210.14782015.14986097.

26. Paschke A, Zaoutis T, Conway P, D X, Keren R. Previous antimicrobial exposure is associated with drug-resistant urinary tract infections in children. Pediatrics 2010; 125:664-672.

27. Gupta K, Hooton TM, Naber KG, Wullt B, Colgan $\mathrm{R}$, MillerLG, et al. International Clinical Practice Guidelines for the Treatment of Acute Uncomplicated Cystitis and Pyelonephritis in Women: a 2010 update by the Infectious Diseases Society of America and the European Society for Microbiology and Infectious Diseases. Clin Infect Dis 2011; 52:e103.

28. Wu C, Lee H, Chen C, Tuan P, Chiu C. High prevalence and antimicrobial resistance of urinary tract infection isolates in febrile young children without localizing signs in Taiwan. J of Microbio, Immunol and Infection 2016; 49:243e248.

29. Caracciolo A, Bettinelli A, Bonato C, Isimbaldi C, Tagliabue A, Longoni L, Bianchetti MG. Antimicrobial resistance among Escherichia coli that cause childhood community-acquired urinary tract infections in Northern Italy. Italian $J$ of Pediatr 2011; 37:3.

30. Cunha BA. New uses for older antibiotics: nitrofurantoin, amikacin, colistin, polymyxin B, doxycycline, and minocycline revisited. Med Clin North Am 2006; 90:1089-1107. 